\title{
Spin relaxation and the Elliott-Yafet parameter in W(001) ultrathin films: Surface states, anisotropy, and oscillation effects
}

\author{
Nguyen H. Long, ${ }^{*}$ Phivos Mavropoulos, ${ }^{\dagger}$ Bernd Zimmermann, Swantje Heers, David S. G. Bauer, \\ Stefan Blügel, and Yuriy Mokrousov \\ Peter Grünberg Institut and Institute for Advanced Simulation, Forschungszentrum Jülich and JARA, D-52425 Jülich, Germany
}

(Received 4 February 2013; revised manuscript received 25 April 2013; published 24 June 2013)

\begin{abstract}
Using first-principles methods based on density-functional theory, we investigate the spin relaxation in $\mathrm{W}(001)$ ultrathin films. Within the framework of the Elliott-Yafet theory, we calculate the spin mixing of the Bloch states and we explicitly consider spin-flip scattering off self-adatoms. At small film thicknesses, we find an oscillatory behavior of the spin-mixing parameter and relaxation rate as a function of the film thickness, which we trace back to surface-state properties. We also analyze the Rashba effect experienced by the surface states and discuss its influence on the spin relaxation. Finally, we calculate the anisotropy of the spin-relaxation rate with respect to the polarization direction of the excited spin population relative to the crystallographic axes of the film. We find that the spin-relaxation rate can increase by as much as $27 \%$ when the spin polarization is directed out of plane, compared to the case when it is in plane. Our calculations are based on the multiple-scattering formalism of the Korringa-Kohn-Rostoker Green-function method.
\end{abstract}

DOI: 10.1103/PhysRevB.87.224420

PACS number(s): 72.25.Rb, 73.50.Bk, 72.25.Ba, 85.75.-d

\section{INTRODUCTION}

In the process of spin relaxation, an excited electron spin population returns to the state of equilibrium that, in nonmagnetic materials, corresponds to zero spin polarization. Despite the fact that the fundamental mechanisms contributing to spin relaxation have been investigated since a long time in various systems, the phenomenon still attracts attention, owing to its importance in spintronics applications, ${ }^{1}$ for example, in giant magnetoresistance ${ }^{2,3}$ or, lately, in the spin Hall effect ${ }^{4-8}$ as well as the inverse spin Hall effect ${ }^{4,6,9}$ that is used to probe spin currents. We mention these examples among a variety of applications in order to stress the practical importance of spin relaxation in thin metallic films, which is part of the motivation for the present work, as a source of loss of spin-mediated information.

There are various mechanisms that can contribute to spin relaxation ${ }^{10-15}$ and in metallic systems they are mostly related to Fermi-surface properties. Although it is clear that in a nonmagnetic metal or metallic film the spin relaxation can be attributed to spin-flip scattering in the presence of the spin-orbit coupling (SOC), many parameters come into play, and one expects the spin-relaxation rate to depend strongly on the film's crystallographic orientation, its thickness and details of the Fermi surface. Of particular importance here ${ }^{16}$ can also be the Rashba states created as a result of spin-orbit interaction acting on the surface bands in the film. ${ }^{17,18}$

In this work, we present a study of free-standing W(001) films with space-inversion symmetry where we consider that the spin-flip scattering is induced by $\mathrm{W}$ adatom impurities. The motivation for restricting the investigation to free-standing films is that we consider them to be generic prototypes for films in layered structures if the material "sandwiching" the filmthe surrounding matrix-is insulating. There is no question that contact at the film surfaces will produce effects that depend on the surrounding matrix, however, we are searching here for physical mechanisms that can in principle still be present in the contact case, even if the details of the band structure are different. In the present analysis, we will face, e.g., oscillatory effects arising from the thickness dependent interaction of surface states that can be replaced by interface states if the films are sandwiched; also, the low dimensionality entails an anisotropy of spin relaxation that should be a quite general effect irrespective of the film contact. Due to the pronounced manifestation of such effects, bcc W(001) films are chosen among $5 d$ transition-metal thin films for a deeper analysis in the present work.

In the presence of structural inversion symmetry, which is the case here in bcc(001) films, the Elliott-Yafet mechanism $^{14,15}$ plays the most important role for spin relaxation. Within this mechanism, the relaxation is realized via spin-orbit mediated spin-flip scattering off impurities at low temperatures and additionally off phonons at higher temperatures. According to Elliott, ${ }^{14}$ in a system with timereversal $^{19}$ and space-inversion symmetries, there are two degenerate Bloch states at each $\mathbf{k}$ point, which can be written as superpositions of up $|\uparrow\rangle$ and down $|\downarrow\rangle$ spinors:

$$
\begin{aligned}
& \Psi_{\mathbf{k}}^{+}(\mathbf{r})=\left[a_{\mathbf{k}}(\mathbf{r})|\uparrow\rangle+b_{\mathbf{k}}(\mathbf{r})|\downarrow\rangle\right] e^{i \mathbf{k} \cdot \mathbf{r}}, \\
& \Psi_{\mathbf{k}}^{-}(\mathbf{r})=\left[a_{-\mathbf{k}}^{*}(\mathbf{r})|\downarrow\rangle-b_{-\mathbf{k}}^{*}(\mathbf{r})|\uparrow\rangle\right] e^{i \mathbf{k} \cdot \mathbf{r}} .
\end{aligned}
$$

Due to the degeneracy, there is an arbitrariness in the selection of $a_{\mathbf{k}}(\mathbf{r})$ and $b_{\mathbf{k}}(\mathbf{r})$, as any superposition of the states in Eq. (1) is also an eigenstate of the Hamiltonian. In practice, the arbitrariness is usually ${ }^{16,20}$ lifted by the demand that the spin expectation value of $\Psi_{\mathbf{k}}^{+}$should be maximal in the $z$ direction, or actually in any chosen direction ${ }^{21,22} \hat{s}$ (which we call the spin-quantization axis, SQA) experimentally defined by the polarization direction of the excited spin population. The spin polarization vector $\mathbf{S}_{\mathbf{k}}^{ \pm}$corresponding to $\Psi_{\mathbf{k}}^{ \pm}$can be calculated via

$$
\mathbf{S}_{\mathbf{k}}^{ \pm}=\frac{1}{2}\left\langle\Psi_{\mathbf{k}}^{ \pm}|\sigma| \Psi_{\mathbf{k}}^{ \pm}\right\rangle,
$$

where $\sigma$ is the vector of Pauli matrices (atomic units with $\hbar=1$ are implied). The two degenerate wave functions at each $\mathbf{k}$ point are orthogonal to each other and have opposite spin expectation values:

$$
\mathbf{S}_{\mathbf{k}}^{-}=-\mathbf{S}_{\mathbf{k}}^{+}
$$


It is straightforward to show the relation of the spin projection along the SQA, $\hat{s} \cdot \mathbf{S}_{\mathbf{k}}^{+}$, to $a_{\mathbf{k}}(\mathbf{r})$ and $b_{\mathbf{k}}(\mathbf{r})$ :

$$
\begin{aligned}
a_{\mathbf{k}}^{2} & :=\int\left|a_{\mathbf{k}}(\mathbf{r})\right|^{2} d^{3} r=\frac{1}{2}+\hat{s} \cdot \mathbf{S}_{\mathbf{k}}^{+}, \\
b_{\mathbf{k}}^{2} & :=\int\left|b_{\mathbf{k}}(\mathbf{r})\right|^{2} d^{3} r=\frac{1}{2}-\hat{s} \cdot \mathbf{S}_{\mathbf{k}}^{+},
\end{aligned}
$$

where we have also introduced the integrals $a_{\mathbf{k}}^{2}$ and $b_{\mathbf{k}}^{2}$. Taking an average over the Fermi surface (FS) (actually Fermi lines in the present two-dimensional case), the Elliott-Yafet parameter (EYP) is obtained as

$$
b^{2}:=\left\langle b_{\mathbf{k}}^{2}\right\rangle_{\mathrm{FS}}=\frac{1}{n\left(E_{\mathrm{F}}\right) V_{\mathrm{BZ}}} \int_{\mathrm{FS}} d k \frac{b_{\mathbf{k}}^{2}}{v_{\mathrm{F}}(\mathbf{k})},
$$

where $v_{\mathrm{F}}(\mathbf{k})$ is the Fermi velocity, $n\left(E_{\mathrm{F}}\right)$ is the density of states at the Fermi level $E_{\mathrm{F}}$, and $V_{\mathrm{BZ}}$ is the Brillouin zone volume.

Momentum scattering events couple the spin-up and -down components of the wave functions at different momenta, allowing for transitions between $\Psi_{\mathbf{k}}^{+}$and $\Psi_{\mathbf{k}^{\prime}}^{-}$and giving rise to spin relaxation. In the Elliott approximation, ${ }^{14}$ the spin-flip probability rate of state $\Psi_{\mathbf{k}}^{+}, P_{\mathbf{k}}^{+-}$, is proportional to the momentum-dependent spin-mixing parameter $b_{\mathbf{k}}^{2}$. As a result, after taking an average over the Fermi surface, the ratio between the spin-relaxation rate $1 / T_{1}$ and momentum-relaxation rate $1 / T_{\mathrm{p}}, T_{1}^{-1} / T_{\mathrm{p}}^{-1}$, is proportional to $b^{2}$. Departing from the Elliott approximation, in which $b^{2}$ is assumed small, one has to take into account the form of the scattering potential $\Delta V$ of the impurity (W adatom in this case) and calculate the scattering $T$ matrix, $T(E)=\Delta V[1-G(E) \Delta V]^{-1}$, where $G(E)$ is the Green function of the unperturbed film. Then, the spin-conserving and spin-flip probability rates are given by the squared matrix elements $P_{\mathbf{k k}^{\prime}}^{++}=2 \pi\left|\left\langle\Psi_{\mathbf{k}}^{+}\left|T\left(E_{\mathrm{F}}\right)\right| \Psi_{\mathbf{k}^{\prime}}^{+}\right\rangle\right|^{2}$ and $P_{\mathbf{k k}^{\prime}}^{+-}=2 \pi\left|\left\langle\Psi_{\mathbf{k}}^{+}\left|T\left(E_{\mathrm{F}}\right)\right| \Psi_{\mathbf{k}^{\prime}}^{-}\right\rangle\right|^{2}$, respectively. Fermi-surface integrals of the scattering probability yield the average spin- and momentum-relaxation rate per $\mathbf{k}$ point:

$$
\begin{gathered}
T_{1}^{-1}(\mathbf{k})=2 T_{\mathrm{sf}}^{-1}(\mathbf{k})=\frac{c}{V_{\mathrm{BZ}}} \int_{\mathrm{FS}} d k^{\prime} \frac{P_{\mathbf{k} \mathbf{k}^{\prime}}^{+-}+P_{\mathbf{k} \mathbf{k}^{\prime}}^{-+}}{v_{\mathrm{F}}\left(\mathbf{k}^{\prime}\right)}, \\
T_{\mathrm{p}}^{-1}(\mathbf{k})=\frac{c}{V_{\mathrm{BZ}}} \int_{\mathrm{FS}} d k^{\prime} \frac{P_{\mathbf{k} \mathbf{k}^{\prime}}^{++}+P_{\mathbf{k k}^{\prime}}^{+-}}{v_{\mathrm{F}}\left(\mathbf{k}^{\prime}\right)},
\end{gathered}
$$

and $\mathbf{k}$-averaged

$$
\begin{gathered}
T_{1}^{-1}=2 T_{\mathrm{sf}}^{-1}=\frac{1}{n\left(E_{\mathrm{F}}\right) V_{\mathrm{BZ}}} \int_{\mathrm{FS}} d k \frac{T_{1}^{-1}(\mathbf{k})}{v_{\mathrm{F}}(\mathbf{k})}, \\
T_{\mathrm{p}}^{-1}=\frac{1}{n\left(E_{\mathrm{F}}\right) V_{\mathrm{BZ}}} \int_{\mathrm{FS}} d k \frac{T_{\mathrm{p}}^{-1}(\mathbf{k})}{v_{\mathrm{F}}(\mathbf{k})}
\end{gathered}
$$

with $c$ the concentration of impurities. The factor 2 in the definition of $T_{1}$ with respect to the spin-flip time $T_{\text {sf }}$ has a historical origin as $T_{1}$ was derived from the full linewidth at half-amplitude of conduction electron spin resonance spectra. ${ }^{23,24}$ In the literature, $T_{1}$ is usually mentioned as the measured quantity, but from the point of view of scattering theory it is more natural to use $T_{\mathrm{sf}}$.

It is clear that the spin-mixing parameter $b^{2}$ reflects the host contribution to the spin relaxation while the spin-relaxation time $T_{1}$ contains also the scattering contribution of the impurity, which of course depends on the type of impurity as well as impurity concentration. In the present work, we will first discuss the spin-mixing parameter to understand general features of spin relaxation in $\mathrm{W}(001)$ thin films. After that, we will investigate the spin-relaxation rate $T_{1}^{-1}$ quantitatively by introducing a $\mathrm{W}$ adatom on one film surface. The scattering matrix is calculated thus for a single $\mathrm{W}$ adatom while the scattering rate is assumed to scale up proportionally to the adatom concentration.

The spin-quantization axis $\hat{s}$ is at first chosen perpendicular to the surface of the films, but later we also examine a variation of the quantization axis revealing anisotropic effects in spin relaxation. The variation of the quantization axis corresponds to an experimental situation where the spin polarization of the injected spin population (or the magnetic field direction in an electron spin resonance experiment) is changed. We should note that the anisotropy discussed here concerns conduction electrons and is different in origin and in nature from the anisotropy of the damping parameter, relaxation, and switching time of the magnetization in ferromagnetic particles that has been discussed in the past. ${ }^{25-27}$

We find that the EYP acquires very large values owing to the Rashba effect at the surface and also exhibits an even-odd oscillation with the thickness of the films following the behavior of the electronic structure of the surface bands; the same is true for the spin-relaxation rate due to scattering off W adatoms. These effects are the subjects of Secs. III and IV. We then present in Sec. V the anisotropy of the EYP and of the spin-relaxation rate with respect to the choice of the SQA. Finally, in Sec. VI, we argue that, as far as the spin relaxation in the surface states is concerned, the mechanism discussed and calculated here should be dominant over, e.g., the Dyakonov-Perel mechanism, prominent in semiconductors or semiconductor heterostructures where Rashba-type of splitting is also present ${ }^{11,12}$ but has a much lower magnitude.

It should be mentioned that $\mathrm{W}(001)$ films have been a subject of intensive theoretical and experimental research for a long time, cf. Refs. $28-30$ and citations therein. It is known that at low temperatures the $\mathrm{W}(001)$ surface undergoes a $c(2 \times 2)$ reconstruction, while the ideal structure is restored at higher temperatures. ${ }^{31,32}$ Additionally, the bcc structure of W(001) films has been extensively studied owing to its multiple applications as a substrate used for deposition. ${ }^{33-36}$ Lately, because of the strong spin-orbit interaction W(001) is used as substrate for ultrathin magnetic films generating a strong Dzyaloshinki-Moriya interaction introducing complex magnetic structures ${ }^{37}$ However, most important for our study are the surface states. Together with $\mathrm{Cu}(111), \mathrm{W}(001)$ is one of the first systems for which surface states were predicted to exist theoretically. ${ }^{38,39}$

\section{METHOD OF CALCULATION}

Our investigation is based on density-functional calculations within the local density approximation. ${ }^{40} \mathrm{We}$ employ the full-potential Korringa-Kohn-Rostoker (KKR) Green function method $^{41}$ with exact treatment of the atomic cell shapes. ${ }^{42} \mathrm{Af}-$ ter a self-consistent full-potential calculation performed within the scalar-relativistic approximation, spin-orbit coupling is added when calculating the Fermi surface properties and the scattering-matrix elements. The formalism for the calculation is given in detail in Ref. 16. A similar formalism ${ }^{43}$ has been 
applied before in Refs. 44 and 45 where the results compared well with experiment.

In brief, the KKR method is based on a site-centered expansion of the Bloch wave function $\Psi_{\mathbf{k}}^{+}$or $\Psi_{\mathbf{k}}^{-}$(we omit the superscript \pm for simplicity in the following equation),

$$
\begin{aligned}
\left(\begin{array}{c}
\Psi_{\mathbf{k}}^{\uparrow}(\mathbf{n}+\mathbf{r}) \\
\Psi_{\mathbf{k}}^{\downarrow}(\mathbf{n}+\mathbf{r})
\end{array}\right)= & \sum_{L}\left(\begin{array}{ll}
R_{L}^{\uparrow \uparrow}(\mathbf{r} ; E) & R_{L}^{\uparrow \downarrow}(\mathbf{r} ; E) \\
R_{L}^{\downarrow \uparrow}(\mathbf{r} ; E) & R_{L}^{\downarrow \downarrow}(\mathbf{r} ; E)
\end{array}\right)\left(\begin{array}{c}
c_{\mathbf{k} L}^{\uparrow} \\
c_{\mathbf{k} L}^{\downarrow}
\end{array}\right), \\
& \times e^{i \mathbf{k} \cdot \mathbf{n}}
\end{aligned}
$$

where $\mathbf{n}$ is a lattice-site vector (with straightforward generalization to multibasis lattices), $\mathbf{r}$ is running in the atomic cell of site $\mathbf{n}$, and $L$ is an angular momentum index. $R_{L}^{s^{\prime} s}(\mathbf{r} ; E)$ are scattering solutions of the Schrödinger equation for the atomic potential (including SOC) in free space at energy $E$ with incoming boundary conditions of spin $s$ and angular momentum $L$, while $c_{\mathbf{k} L}^{s}(E)$ are energydependent expansion coefficients found from the KKR secular equation. ${ }^{16}$ To clarify the connection of the above representation with Eq. (1), we note that $\Psi_{\mathbf{k}}^{\uparrow}(\mathbf{r})=a_{\mathbf{k}}(\mathbf{r}) e^{i \mathbf{k} \cdot \mathbf{r}}$ and $\Psi_{\mathbf{k}}^{\downarrow}(\mathbf{r})=b_{\mathbf{k}}(\mathbf{r}) e^{i \mathbf{k} \cdot \mathbf{r}}$. For the calculation of the scattering properties, we calculate the spin-dependent Green function $G_{s s^{\prime}}\left(\mathbf{r}, \mathbf{r}^{\prime} ; E\right)$ in an analogous expansion as the Bloch wave function with coefficients $G_{L s ; L^{\prime} s^{\prime}}^{\mathbf{n n}}(E)$ that are found for the system with impurity by means of a Dyson equation using the host-crystal Green function as a reference. Two types of freeelectron scattering matrices are defined corresponding to the difference $\Delta V_{s s^{\prime}}(\mathbf{r})$ between impurity and host potential (including SOC), $\Delta_{L L^{\prime}}^{s s^{\prime}}(E)=\sum_{s^{\prime \prime} s^{\prime \prime \prime}} \int d^{3} r\left(R_{L}^{s^{\prime \prime} s}\right)^{*} \Delta V_{s^{\prime \prime} s^{\prime \prime \prime}} R_{L^{\prime}}^{\mathrm{imp} ; \mathrm{s}^{\prime \prime \prime} \mathrm{s}^{\prime}}$ and $\Delta t_{L L^{\prime}}^{s s^{\prime}}(E)=\sum_{s^{\prime \prime} s^{\prime \prime \prime}} \int d^{3} r R_{L}^{s^{\prime \prime} s^{\prime}} \Delta V_{s^{\prime \prime} s^{\prime \prime \prime}} R_{L^{\prime}}^{\text {imp;s"'s }}$ with the superscript "imp" indicating the impurity solution. The scattering matrix elements off the impurity at site n then are calculated as $\left\langle\Psi_{\mathbf{k}}^{+}\left|T\left(E_{\mathrm{F}}\right)\right| \Psi_{\mathbf{k}^{\prime}}^{+}\right\rangle=\sum\left(c_{\mathbf{k} L}^{s}\right)^{*}\left[\Delta_{L L^{\prime}}^{s s^{\prime}}+\right.$ $\left.\Delta_{L L^{\prime \prime}}^{s s^{\prime \prime}} G_{L^{\prime \prime} s^{\prime \prime} ; L^{\prime \prime \prime} s^{\prime \prime \prime}}^{\mathbf{n n}} \Delta t_{L^{\prime \prime \prime} L^{\prime}}^{s^{\prime \prime \prime} s^{\prime}}\right] c_{\mathbf{k}^{\prime} L^{\prime}}^{s^{\prime}}$ where the summation is implied to run over all angular momentum and spin indices, and analogously for $\left\langle\Psi_{\mathbf{k}}^{+}\left|T\left(E_{\mathrm{F}}\right)\right| \Psi_{\mathbf{k}^{\prime}}^{-}\right\rangle$. A generalization to a scattering cluster containing the perturbed neighbors of the impurity is straightforward and done in our calculations in order to account correctly for the perturbed charge density.

An angular momentum cutoff of $l_{\max }=3$ is taken. We use the experimental lattice constant of bcc tungsten of $a=$ $3.165 \AA$. For an interpretation of our results and a separation of causes we perform in some cases numerical experiments by switching on and off the spin-orbit coupling in the system. The $\mathrm{W}$ adatom impurity is assumed to reside on the fourfold hollow position on the surface. Its electronic structure is treated using the Jülich KKR impurity-embedding code (KKRIMP) ${ }^{46}$ which enables us to treat the charge and spin-density self-consistently including the perturbation of the nearest neighbors of the impurity. Lattice relaxations are not taken into account.

\section{EVEN-ODD EFFECT IN SPIN-MIXING PARAMETER AND SPIN-RELAXATION RATE}

We first present our results on an unexpected even-odd oscillatory variation that we observed in the Elliott-Yafet parameter of the film and the spin-relaxation rate due to a $\mathrm{W}$ adatom with respect to the number of layers in the $\mathrm{W}(001)$ film. To start the discussion, we take a look at the

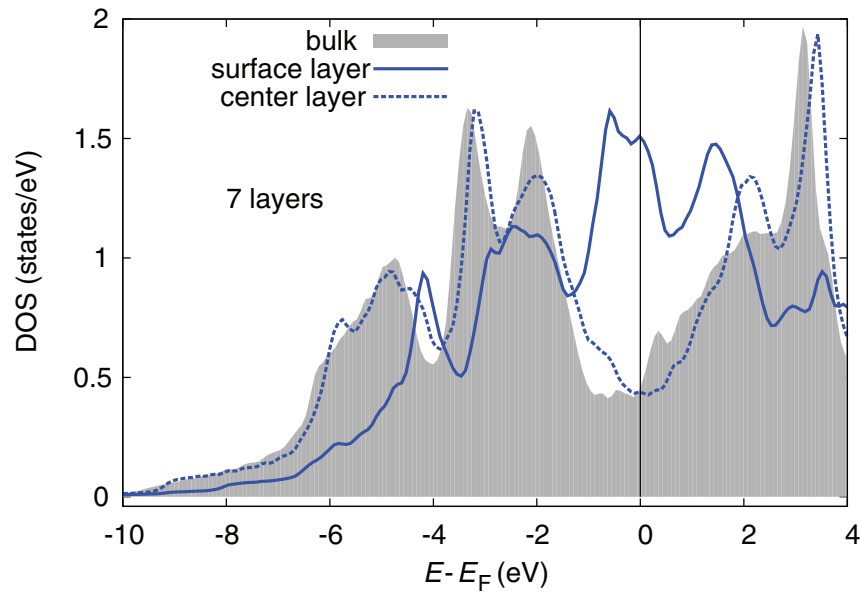

FIG. 1. (Color online) The atom-resolved density of states (DOS) of the surface and of the central layer of a 7-layer thick W(001) film in comparison to the DOS of bulk W shown as a grey-shaded area.

comparison of the density of states (DOS), including SOC, of $\mathrm{W}$ bulk and a typical representative of the films studied in the following-a 7-layer W(001) film-shown in Fig. 1. Characteristic of a transition metal with bcc structure, the bulk DOS shows a bimodular behavior with a dip in the middle separating bonding from antibonding states, which for $\mathrm{W}$ lies at the Fermi energy. This structure of the DOS is also clearly preserved in the center of the thin W(001) film. In contrast, the local DOS in the surface atomic layer of the film displays a peak at $E_{\mathrm{F}}$, which can be attributed to the presence of surface states. Obviously, this should result in a significant surface contribution to the Fermi-surface-dependent quantities and, in particular, the Elliott-Yafet parameter. The DOS of W(001) films of different thickness, considered later, are very similar to those in the case of a 7-layer film.

Next, we choose the spin-quantization axis perpendicular to the film $(\hat{s}||[001])$ and calculate the Fermi surfaces and the distribution of the spin-mixing parameter $b_{\mathbf{k}}^{2}$ for W(001) films of varying thickness. The results are presented in Fig. 2. The most important feature for our study are the surface states. These are indicated as "S1" and "S2," and pointed at by arrows in the vicinity of the $\bar{M}$ point on the Fermi surface. S1 and $\mathrm{S} 2$ are present at all film thicknesses and we will discuss them in more detail below. Here we merely note for clarity that the S2-line merges into bulklike states at its two ends (indicated by two small circles in Fig. 2), while S1 does not; also, that S1 appears as a double line due to SOC-induced Rashba splitting and due to the interaction between the two film surfaces, while S2 appears as a single line because its partner is higher than $E_{\mathrm{F}}$ in energy.

The importance of the surface states for spin relaxation can be ascertained by looking at the Fermi-surface-integrated Elliott-Yafet parameter $b^{2}$ [see Eq. (6)] as a function of film thickness, additionally decomposed into surface and bulk contributions and presented in the top panel of Fig. 3. The latter decomposition was performed by integrating in Eq. (6) over only the surface-state part or only the bulk-state part of the Fermi surface, while keeping the denominator $n\left(E_{\mathrm{F}}\right)$ fixed at the value of the total density of states. It is striking that for all considered film thicknesses the overall contribution of the 

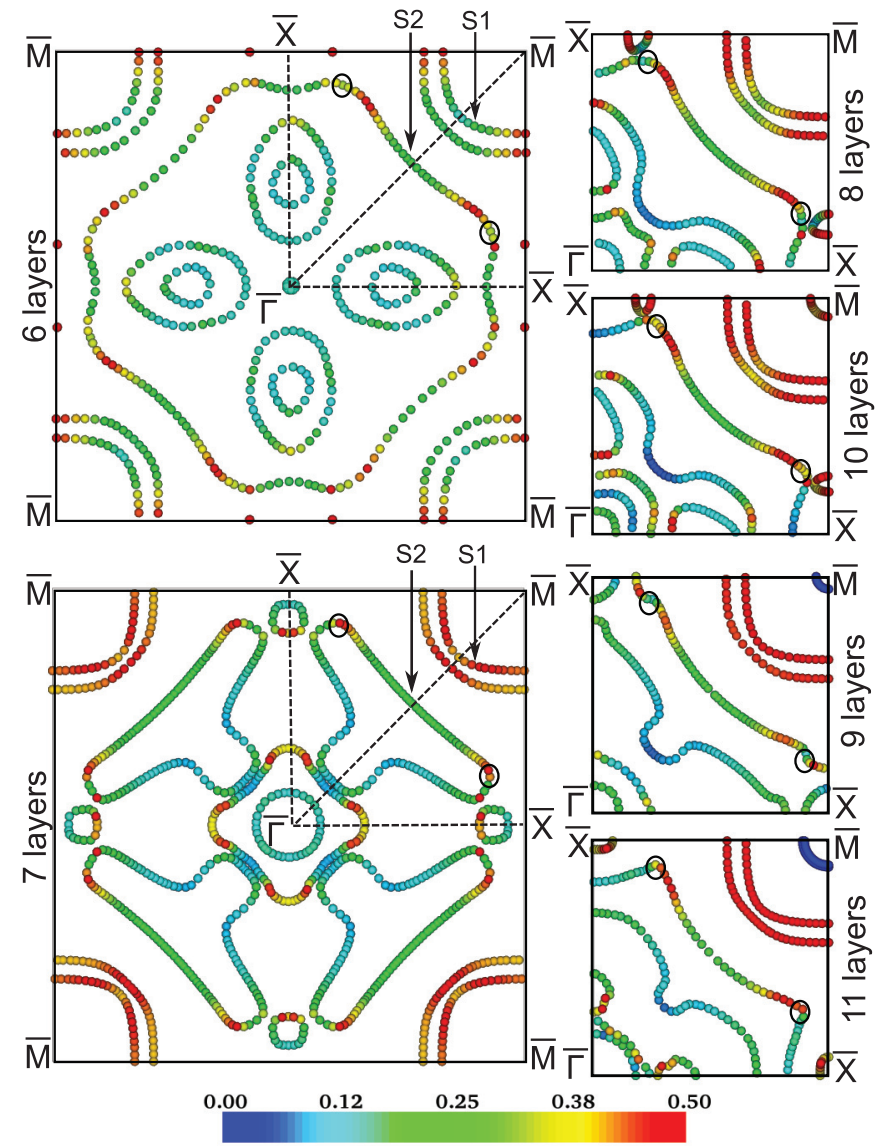

FIG. 2. (Color online) Fermi surfaces calculated including SOC and distribution of the spin-mixing parameter $b_{\mathbf{k}}^{2}$ (color of the Fermi surface points) for $\mathrm{W}(001)$ films of various thickness. The Fermi surfaces are grouped into those for films with even number (upper group) and odd number (lower group) of layers. The surface states S1 and S2 are marked with arrows. Small circles indicate where S2 merges into the bulklike states.

surface states to the EYP is comparable to, or even larger than, the bulk contribution. It is well known that the amplitude of the surface states drops exponentially as a function of distance from the surface. Therefore, in the limit of large film thickness, the relative contribution of the surface states decreases and the bulk contribution becomes prominent. However, in the ultrathin films studied here (maximum 12-layers thickness), the surface states are more like quantum-well states and do not fully decay in the center of the film. Additionally, there are two more reasons why the bulk limit is not reached fast. First, the density of bulklike states at $E_{\mathrm{F}}$ is at a minimum as we saw in Fig. 1, thus the bulk contribution to $b^{2}$ sets in only slowly. Second, the Fermi surface of bulk W is rather complex with the value of $b_{\mathbf{k}}^{2}$ strongly varying over it (see, e.g., Ref. 21), thus many film layers are needed to achieve the equivalent of a good resolution in the [001] direction of the bulk Brillouin zone that would yield the bulk limit $b^{2}=0.065 .{ }^{21}$ Surprisingly, as we observe from Fig. 3, the contribution of the surface state S2 and correspondingly the total EYP displays a pronounced even-odd oscillation as a function of film thickness up to 10 layers.

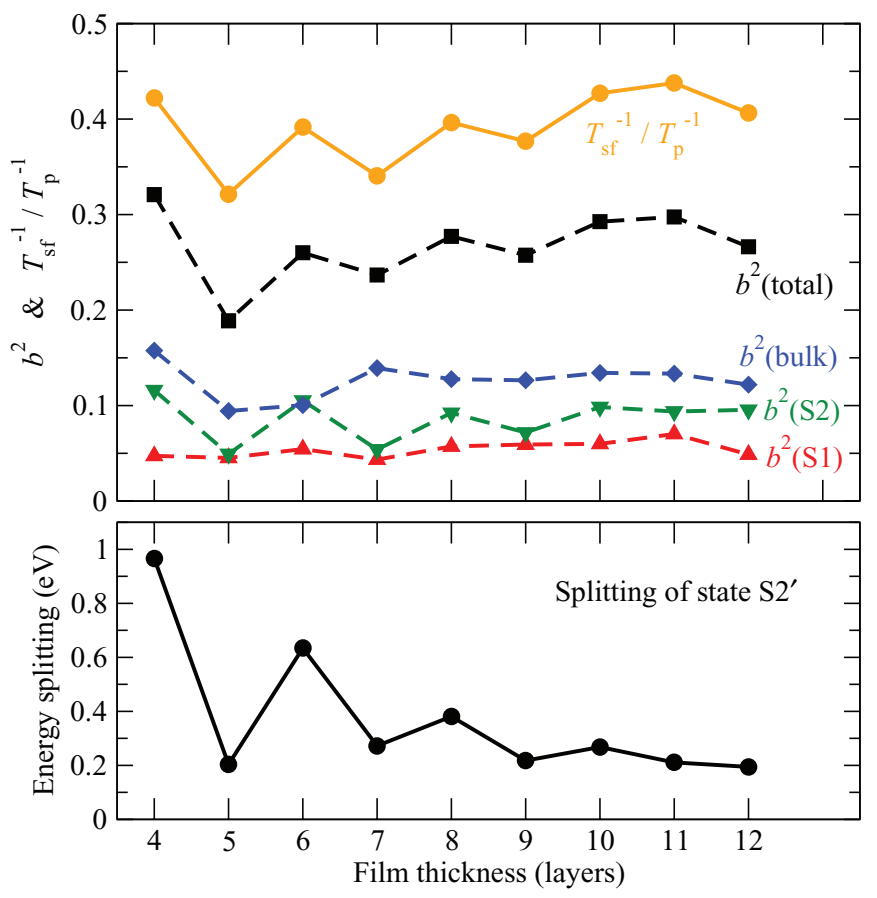

FIG. 3. (Color online) (Top) The dashed lines show the thickness dependence of the Elliott-Yafet parameter in W(001) thin films with the SQA perpendicular to the film. The total EYP (squares) is decomposed into contributions from the surface states S1 and S2 (triangles) and from the bulk states (diamonds) of the film. The full line (circles) shows the ratio between spin-flip rate and momentum-relaxation rate, $T_{\mathrm{sf}}^{-1} / T_{\mathrm{p}}^{-1}$ for the case of self-adatom scattering [W adatom on $\mathrm{W}(001)]$. The even-odd effect is evident in the ratio following very closely the oscillation of the Elliott-Yafet parameter. (Bottom) Splitting of the state S2' (without SOC) due to the interaction between the two surface states of opposite surfaces (see Fig. 4 for the band structure and for the position where the splitting of S2' is calculated). The oscillatory behavior of the splitting as function of film thickness correlates with the behavior of the EYP and spin-flip rate. The lines are guides to the eye.

In the distribution of $b_{\mathbf{k}}^{2}$ on the Fermi surface for several film thicknesses, presented in Fig. 2, we see that $b_{\mathbf{k}}^{2}$ exhibits large variations in magnitude, both as a function of the position at the Fermi surface as well as the film thickness. We also clearly observe that among all states at the Fermi surface the largest spin-mixing occurs in the surface states S1 and S2.

We will now analyze the oscillation with film thickness starting from the band structure and symmetry properties of the surface states. It is useful to compare the calculation of the surface band structure with and without SOC for two reasons. First, the surface states suffer a splitting that is partly due to SOC and partly due to the interaction between the two surfaces of the film, thus the calculation without SOC helps to distinguish between the two mechanisms. Second, an analysis of the penetration of the surface states into the bulk region is easier in the absence of SOC because of particular symmetry arguments that do not hold in the presence of SOC, even if the qualitative form of the wavefunctions in this respect is similar. We name the surface states $\mathrm{S}^{\prime}$ and $\mathrm{S}^{\prime}{ }^{\prime}$ where the primed indices are used to distinguish the case without $\mathrm{SOC} ; \mathrm{S}^{\prime}$ and 

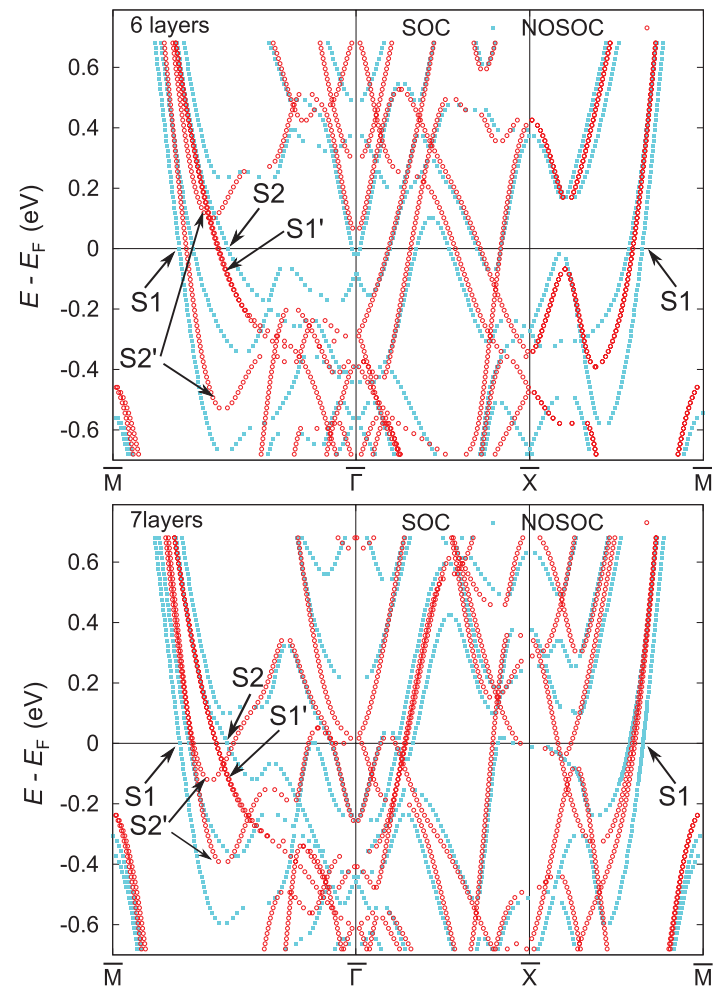

FIG. 4. (Color online) The band structure of 6- and 7-layer $\mathrm{W}(001)$ films with (filled blue squares) and without (red open circles) spin-orbit coupling. Arrows mark the surface states S1 and $\mathrm{S} 2$ corresponding to the calculation with $\mathrm{SOC}$ and $\mathrm{S}^{\prime}$ and $\mathrm{S} 2^{\prime}$ corresponding to the calculation without SOC. An even-odd effect in the thickness-dependent splitting of $\mathrm{S}^{\prime}$ is responsible for the even-odd effect in the EYP.

$\mathrm{S} 2^{\prime}$ are mixed with each other under the action of the SOC Hamiltonian to produce S1 and S2.

We consider the band structure of $\mathrm{S}^{\prime}{ }^{\prime}$ and $\mathrm{S} 2^{\prime}$ along the $\overline{\Gamma \mathrm{M}}$ diagonal of the Brillouin zone (red open circles in Fig. 4). $\mathrm{S}^{\prime}{ }^{\prime}$ and $\mathrm{S} 2^{\prime}$ comprise mainly $d$ orbitals. We set a coordinate system with $x$ and $y$ along the [100] and [010] directions in the surface and $z$ normal to the surface. We distinguish two types of $d$ orbitals with respect to their reflection properties about the diagonal [110] ( $\overline{\Gamma \bar{M}})$ : even $\left[\frac{1}{\sqrt{2}}\left(d_{x z}+d_{y z}\right), d_{x y}\right.$, and $\left.d_{z^{2}}\right]$ and odd $\left[\frac{1}{\sqrt{2}}\left(d_{x z}-d_{y z}\right)\right.$ and $\left.d_{x^{2}-y^{2}}\right]$. Staying on the high-symmetry line $\mathbf{k} \in \overline{\Gamma \mathrm{M}}$, the Bloch states $\Psi_{\mathbf{k}}(\mathbf{r})$ derived from odd orbitals show nodes along the [110] diagonal, which has a consequence of a high in-plane kinetic energy, not leaving enough energy for penetration into the bulk region. Therefore the odd- $d$-orbital surface states of the opposite surfaces couple very weakly to each other and show an almost vanishing splitting already at small film thicknesses. On the other hand, the even states, relieved from this nodal structure, have less in-plane kinetic energy and thus enough energy to penetrate into the bulk region and hybridize with their likes of the opposite surface; then the resulting hybrids show a splitting even at larger thicknesses. This parity-dependence of the surface states was observed in the past by Mattheiss and Hamann. ${ }^{30}$ The consequence of this can be seen, e.g., in Fig. 4 for the bands without spin-orbit coupling along $\overline{\Gamma \mathrm{M}}$ where the $\mathrm{S} 2$ ' bands show a large splitting whereas the bands $\mathrm{S}^{\prime}$ show an almost complete degeneracy.

The even-odd effect of the EYP of state S2 can now be traced back to surface state $\mathrm{S} 2^{\prime}$. The aforementioned splitting of $S 2^{\prime}$ due to the interaction between the opposite surfaces exhibits an even-odd behavior: for an even number of layers the splitting is large, for odd it is considerably smaller due to an oscillation in the coupling. This can be seen in Fig. 3 (bottom panel) where the splitting of S2' has been calculated at the position indicated by arrows in Fig. 4. One immediately recognizes the striking correlation between the oscillations in the splitting of $\mathrm{S2}^{\prime}$ and in the EYP shown in the same figure. We should comment that the origin of the even-odd effect in the coupling could not be fully explained by the symmetry properties of the wave functions. However, it is clear that owing to the different inversion symmetry center in evenand odd-layer films, the overlap between the opposite-side surface states is different, which contributes to the even-odd effect.

Including the SOC, the even-odd dependence of the energy splitting will have a profound effect on the spin-mixing parameter, since the energy distance between states affects crucially the value of $b_{\mathbf{k}}$ in Eq. (1). To justify this statement, we remind the reader of Elliott's perturbative expansion: ${ }^{14}$ for the $n$th band a summation over all other bands $n^{\prime} \neq n$ should be performed, reading $b_{n \mathbf{k}}^{2}=\sum_{n^{\prime}}\left|\left\langle\Psi_{n \mathbf{k}}^{0}\left|\xi(L S)^{\uparrow \downarrow}\right| \Psi_{n^{\prime} \mathbf{k}}^{0}\right\rangle\right|^{2} /\left(E_{n \mathbf{k}}^{0}-E_{n^{\prime} \mathbf{k}}^{0}\right)^{2}$ in first-order perturbation theory, where the superscript 0 refers to the wave functions and eigenstates without SOC, $\xi$ is the SOC strength, and $(L S)^{\uparrow \downarrow}$ is the spin-flip part of SOC operator. Since the energy difference of the states appears in the denominator (in all orders of the perturbation expansion), changing the energy splitting will strongly affect the value of $b_{\mathbf{k}}^{2}$. Thus emerges the correlation between the splitting of $\mathrm{S}^{\prime}{ }^{\prime}$ without SOC and the EYP with SOC in Fig. 3.

Now we investigate the spin relaxation due to the $\mathrm{W}$ adatom impurities located on one film surface. In the upper panel of Fig. 3, we also show the ratio between the spin-flip rate and momentum-relaxation rate, $T_{\mathrm{sf}}^{-1} / T_{\mathrm{p}}^{-1}$, as a function of the film thickness. We see that the magnitude of the ratio is of the same order as the Elliott-Yafet parameter and that the oscillatory behavior of the two quantities is clearly correlated, even though Elliott's approximate relation $T_{\mathrm{sf}}^{-1} / T_{\mathrm{p}}^{-1} \approx 4 b^{2}$ does not hold. The deviation from Elliott's approximation is not surprising since it holds under the assumptions that $b^{2}$ is small and that the scattering is weak enough to be described within first-order perturbation theory. In any case the high values of $T_{\mathrm{sf}}^{-1} / T_{\mathrm{p}}^{-1}$, of the order of $0.3-0.4$, show that approximately every third scattering event includes a spin-flip. This high spin-flip rate is certainly related with the fact that a $\mathrm{W}$ adatom introduces an additional contribution to spin-flip scattering via its internal spin-orbit coupling. It can be remarked that the ratio between spin-flip rate and momentum-relaxation rate does not depend on the impurity concentration, but the values of the two quantities separately do. In order to have a quantitative estimate, the impurity concentration $c$ in Eqs. (7) and (8) is set to be $1 \%$. This gives us, for example, a value of the spin-relaxation rate of $T_{1}^{-1}=2 T_{\mathrm{sf}}^{-1}=9.57 \mathrm{ps}^{-1} / \mathrm{at} \%$ and momentum-relaxation rate of $T_{\mathrm{p}}^{-1}=11.35 \mathrm{ps}^{-1} / \mathrm{at} \%$ for a 10-layer $\mathrm{W}(001)$ thin film by scattering off $\mathrm{W}$ adatoms. We will return to the spin relaxation rate in Sec. V. 


\section{RASHBA-CHARACTER OF SURFACE STATES}

This section is dedicated to the investigation of the Rashba effect at the W(001) surface. The essence of the Rashba effect at surfaces lies in a crystal-momentum dependent splitting of surface states due to the presence of spin-orbit coupling together with the structural inversion asymmetry caused by the surface. ${ }^{47,48}$ As was first observed experimentally in $\mathrm{Au}(111)$ by LaShell et al. ${ }^{17}$ and then theoretically explained by Henk and co-workers, ${ }^{18}$ the surface states of $\mathrm{Au}(111)$ display a SOC-derived dispersion relation very similar to that of the two-dimensional electron gas (2DEG) described by a two-band Rashba model. ${ }^{47}$ One signature of the Rashba effect is a Fermi surface consisting of two concentric rings with the spin polarization perpendicular to the group velocity but pointing in opposite directions at each of the rings. We will refer to the resulting k-space spin texture as spin-polarization field in the following (which is closely related to the spin-orbit field defined, e.g., in Ref. 12).

The Rashba splitting results in a degeneracy lifting where the pairing implied by Eq. (1) does not hold any more. Since the effect originates in the broken inversion symmetry at the surface, it can be captured computationally by treating halfinfinite systems or by breaking the symmetry between two surfaces of a finite symmetric slab via deposition on a substrate or adsorption of a monolayer of a different material on one surface of the slab, e.g., a $\mathrm{Cu}$ monolayer on an $\mathrm{Au}$ film ${ }^{49}$ or $\mathrm{H}$ adsorption on W(110). ${ }^{50}$ Here, we follow an alternative approach, keeping the inversion symmetry but projecting out the appropriate state from the doubly degenerate subspace defined in Eq. (1). ${ }^{51}$

We introduce an intuitive view of our approach by means of a schematic energy level diagram in Fig. 5 (top). We first imagine the film in the limit of infinite thickness, with the states of each surface not interacting with the opposite surface. Then, a Rashba splitting occurs due to SOC. Fixing a k point on the surface band, the splitting results in spin-polarized levels $|\mathrm{L} \uparrow\rangle$ and $|\mathrm{L} \downarrow\rangle$ on the left surface (indicated by $\mathrm{L}$ ) and similarly $|\mathrm{R} \uparrow\rangle$ and $|\mathrm{R} \downarrow\rangle$ on the right surface. The Rashba splitting $\Delta$ is the same in the two surfaces but the order with respect to energy in which the levels of spin polarization $\uparrow$ and $\downarrow$ occur is reversed in the two surfaces because of the opposite direction of the surface normal, according to standard Rashba theory. Reducing the film thickness, the left and right states interact via a hopping $t$ (let us assume $t \ll \Delta$ ) and hybrid states are formed with bonding (B) and antibonding (A) nature: $|\mathrm{B} \uparrow\rangle \approx|\mathrm{L} \uparrow\rangle+\frac{\mathrm{t}}{\Delta}|\mathrm{R} \uparrow\rangle,|\mathrm{A} \uparrow\rangle \approx-\frac{\mathrm{t}}{\Delta}|\mathrm{L} \uparrow\rangle+|\mathrm{R} \uparrow\rangle$. Thus $|\mathrm{B} \uparrow\rangle$ is localized more on the left surface and $|\mathrm{A} \uparrow\rangle$ more on the right surface. Analogously, hybrids $|\mathrm{B} \downarrow\rangle$ and $|\mathrm{A} \downarrow\rangle$ are formed. A calculation of the film band structure finds linear combinations of the degenerate bonding $\alpha|\mathrm{B} \uparrow\rangle+\beta|\mathrm{B} \downarrow\rangle$ as well as antibonding $\alpha|\mathrm{A} \uparrow\rangle+\beta|\mathrm{A} \downarrow\rangle$ hybrids; such linear combinations are basically the degenerate wave functions $\Psi_{\mathbf{k}}^{ \pm}$ described by Eq. (1) in the particular case of surface states. Returning to the $\Psi_{\mathbf{k}}^{ \pm}$notation of Eq. (1), it has then to be decided which particular linear combination is of interest for the physics of the problem at hand. For instance, for the calculation of the EYP in the previous section the linear combinations were chosen so that the spin expectation value along the $z$ direction was maximized. Here, on the other hand,
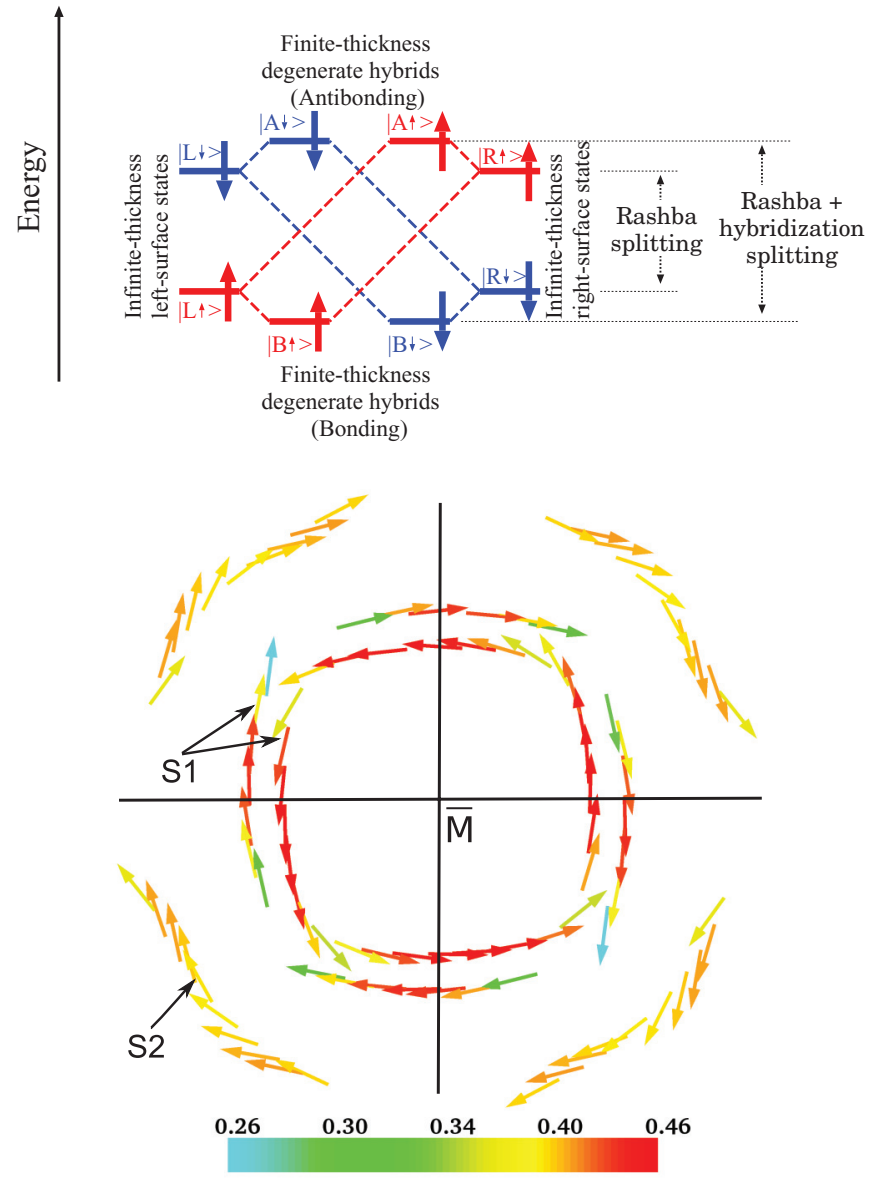

FIG. 5. (Color online) (Top) Schematic diagram showing the energy levels of the Rashba states at the film surfaces and their hybridization when the film thickness is finite and the states of opposite surfaces interact. The full lines represent the energy levels before and after hybridization and the dashed lines show which level pairs are hybridized. (Bottom) The spin polarization of the surface states around the $\bar{M}$ point of an 11-layer W(001) film. The direction of the spin polarization is given by arrows, while its magnitude is given by means of a color code of the arrows. Surface states S1 and $\mathrm{S} 2$ are marked.

we want to choose constants $\alpha_{\mathbf{k}}$ and $\beta_{\mathbf{k}}$ (not depending on $\mathbf{r}$ ) in such a way that the combination $\alpha_{\mathbf{k}} \Psi_{\mathbf{k}}^{+}+\beta_{\mathbf{k}} \Psi_{\mathbf{k}}^{-}$resembles as much as possible the Rashba states of the infinite-thickness film, i.e., in a way that the wave functions $|\mathrm{B} \uparrow\rangle,|\mathrm{B} \downarrow\rangle,|\mathrm{A} \uparrow\rangle$, and $|\mathrm{A} \downarrow\rangle$ of Fig. 5 are retrieved. For this reason we pick one surface, say the left, and we find the linear combination that maximizes the spin expectation value within the particular surface atomic layer and call the resulting wave function $\Psi_{\mathbf{k}}^{\max , \mathrm{L}}=\alpha_{\mathbf{k}} \Psi_{\mathbf{k}}^{+}+\beta_{\mathbf{k}} \Psi_{\mathbf{k}}^{-}$. We thus define the polarization in the surface layer as

$$
\left\langle S_{\mathbf{k}}^{i}\right\rangle_{\text {surf }}=\frac{1}{2} \int_{\text {surf.layer }}\left[\Psi_{\mathbf{k}}^{\max , \mathrm{L}}(\mathbf{r})\right]^{\dagger} \sigma^{i} \Psi_{\mathbf{k}}^{\max , \mathrm{L}}(\mathbf{r}) d^{3} r,
$$

$i \in\{x, y, z\}$, and demand that $\alpha_{\mathbf{k}}$ and $\beta_{\mathbf{k}}$ are such that $\left|\left\langle\mathbf{S}_{\mathbf{k}}\right\rangle_{\text {surf }}\right|=\sqrt{\left\langle S_{\mathbf{k}}^{x}\right\rangle_{\text {surf }}^{2}+\left\langle S_{\mathbf{k}}^{y}\right\rangle_{\text {surf }}^{2}+\left\langle S_{\mathbf{k}}^{z}\right\rangle_{\text {surf }}^{2}}$ is maximized. Then we observe that $\Psi_{\mathbf{k}}^{\max , \mathrm{L}}$ has both the spin and charge density more localized on the left surface (numerical result not shown here explicitly). Since the film potential in our 
calculation is still inversion-symmetric, $\Psi_{\mathbf{k}}^{\max , \mathrm{L}}=\alpha_{\mathbf{k}} \Psi_{\mathbf{k}}^{+}+$ $\beta_{\mathbf{k}} \Psi_{\mathbf{k}}^{-}$has an orthogonal degenerate partner, $\Psi_{\mathbf{k}}^{\max , \mathrm{R}}=$ $\alpha_{-\mathbf{k}}^{*} \Psi_{\mathbf{k}}^{-}-\beta_{-\mathbf{k}}^{*} \Psi_{\mathbf{k}}^{+}$, which is more localized on the opposite surface and has the opposite spin expectation value. We say "more localized" and not "completely localized" because $\Psi_{\mathbf{k}}^{\max , \mathrm{L} / \mathrm{R}}$ are still left-right hybrids but each has higher amplitude on its representative surface. Thus we have conveniently separated the degenerate surface states in a way that they naturally evolve into the single surface state case in the limit of infinite film thickness. It should be stressed here that maximizing $\left|\left\langle\mathbf{S}_{\mathbf{k}}\right\rangle_{\text {surf }}\right|$ is only the means of choosing a reasonable approximation to the single-surface state. However, we analyze the resulting spin-polarization field by calculating the spin expectation value of $\Psi_{\mathbf{k}}^{\max , \mathrm{L}}$ over all layers, $\left\langle\mathbf{S}_{\mathbf{k}}\right\rangle=\frac{1}{2}\left\langle\Psi_{\mathbf{k}}^{\max , \mathrm{L}}|\boldsymbol{\sigma}| \Psi_{\mathbf{k}}^{\max , \mathrm{L}}\right\rangle$.

In Fig. 5, we present our results on the spin-polarization field, represented by arrows, for an 11-layer W(001) film. The figure is focused on the area of reciprocal space around the $\overline{\mathrm{M}}$ point, i.e., partly outside the first Brillouin zone, to show the contours that correspond to the surface states S1 and S2. The starting point of each arrow corresponds to a $\mathbf{k}$ point on the Fermi surface, the direction of the arrow to the direction of $\left\langle\mathbf{S}_{\mathbf{k}}\right\rangle$, and the color code to its magnitude. The out-of-plane component of spin polarization is found to vanish.

The spin-polarization field of surface state S1 (two concentric rings around $\overline{\mathrm{M}}$ ) reminds one of the "pure" Rashba states at the $\mathrm{Au}(111)$ surface, in the sense that $\left\langle\mathbf{S}_{\mathbf{k}}\right\rangle$ is in-plane and almost perpendicular to $\mathbf{k}-\mathbf{k}_{0}$, where $\mathbf{k}_{0}$ is the ring center (here the $\overline{\mathrm{M}}$-point; in the Rashba model for $\mathrm{Au}(111)$ the $\bar{\Gamma}$ point). More precisely, we observe that $\left\langle\mathbf{S}_{\mathbf{k}}\right\rangle$ is perpendicular to the group velocity, i.e., $\left\langle\mathbf{S}_{\mathbf{k}}\right\rangle$ is along the Fermi-surface tangent, as expected from the Rashba model. As for the spin-polarization field of the state $S 2$, first we should remind the reader that S2 merges with the bulk-state continuum (see Fig. 2), excluded from the plot, which leads to the fact that the shown contour in Fig. 5 ends seemingly abruptly. Second, S2 also has a partner with opposite direction of the spin-polarization field, which lies higher in energy (see the band-structure in Fig. 4). $\left\langle\mathbf{S}_{\mathbf{k}}\right\rangle$ in $\mathrm{S} 2$ is also in-plane and perpendicular to the group velocity.

Finally, it should be noted that the magnitude of the spin-polarization fields, $\left|\frac{1}{2}\left\langle\Psi_{\mathbf{k}}(\mathbf{r})|\sigma| \Psi_{\mathbf{k}}(\mathbf{r})\right\rangle\right|$ cannot reach the maximal value $\frac{1}{2}$, except perhaps in the pure Rashba model, since an interaction with other states at other energies at the same $\mathbf{k}$ via the spin-orbit operator will always be present and will reduce the value. To view this from a different perspective, the states $\Psi_{\mathbf{k}}^{ \pm}(\mathbf{r})$ and their linear combination $\Psi_{\mathbf{k}}^{\max }(\mathbf{r})=\alpha_{\mathbf{k}} \Psi_{\mathbf{k}}^{+}(\mathbf{r})+\beta_{\mathbf{k}} \Psi_{\mathbf{k}}^{-}(\mathbf{r})$ result in a noncollinear spindensity $\frac{1}{2} \Psi_{\mathbf{k}}(\mathbf{r})^{\dagger} \boldsymbol{\sigma} \Psi_{\mathbf{k}}(\mathbf{r})$ that can be brought in a diagonal form only in an r-dependent reference frame; but in order to achieve $\left|\left\langle\mathbf{S}_{\mathbf{k}}\right\rangle\right|=\frac{1}{2}$, this reference frame would have to be independent of $\mathbf{r}$.

\section{ANISOTROPY OF THE ELLIOTT-YAFET PARAMETER AND OF THE SPIN-FLIP SCATTERING RATE OFF ADATOMS}

We now examine the anisotropy of the spin-relaxation rate with respect to the polarization direction of the injected spin population (i.e., the SQA $\hat{s}$ ) relative to the crystallographic axes. For bulk systems, we have already discussed the effect in Ref. 21, pointing out that the reduced symmetry of thin films will clearly play a role in this phenomenon. To summarize the origin of the effect, ${ }^{21}$ even though the spin-orbit operator $\mathbf{L} \cdot \mathbf{S}$ is independent of the SQA, its matrix elements are not. Particularly relevant here are the matrix elements of the spinflip SOC $(L S)^{\uparrow \downarrow}, \frac{1}{2}\left(L_{+} S_{-}+L_{-} S_{+}\right)$, that are responsible for the spin-mixing parameter $b_{\mathbf{k}}^{2}$ and for the spin-flip transitions in general. Due to this dependence on the SQA, and considering the crystallographic symmetry of the W(001) film, we expect at least three inequivalent directions of $\hat{s}$ for which the spinrelaxation rate $T_{1}^{-1}$ will become extremal: perpendicular to the film, in-plane in the [100] direction and in-plane in the [110] direction. Comparing the values of $T_{1}^{-1}(\hat{s})$ in all directions, we obtain the definition of the anisotropy

$$
\mathcal{A}\left[T_{1}^{-1}\right]=\left[\max _{\hat{s}} T_{1}^{-1}(\hat{s})-\min _{\hat{s}} T_{1}^{-1}(\hat{s})\right] / \min _{\hat{s}} T_{1}^{-1}(\hat{s}),
$$

which is a somewhat different quantity compared to $\mathcal{A}\left[b^{2}\right]$ introduced in Ref. 21, where we had $b^{2}(\hat{s})$ in the place of $T_{1}^{-1}(\hat{s})$ implying the Elliott approximation. We wish to point out here that not only the spin-flip rate but also the spin-conserving rate depends on the SQA, and that part of the anisotropy comes from the SQA dependence of $b_{\mathbf{k}}^{2}$ in the Bloch states, while another part comes from the spin-flip scattering off the impurity potential. The total scattering rate (spin flip plus spin conserving) defined by Eqs. (8) and (10) is independent of $\hat{s}$.

In Fig. 6, we show in a color code the value of $b^{2}(\hat{s})$ and $T_{1}^{-1}(\hat{s})$ as a function of the direction $\hat{s}$ on the unit sphere for a 10-layer $\mathrm{W}(001)$ film with a $\mathrm{W}$ adatom as scatterer. The

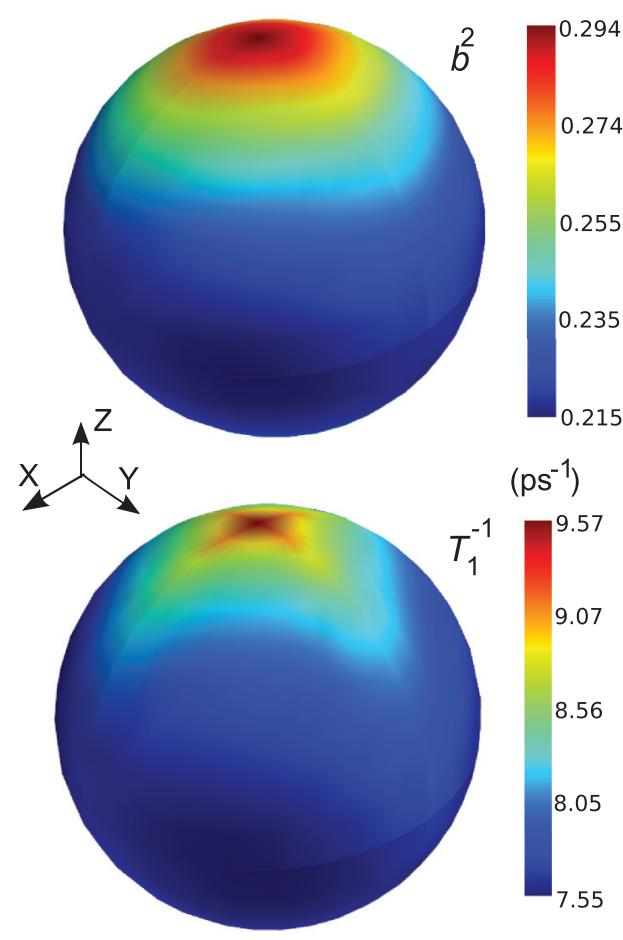

FIG. 6. (Color online) Value of $b^{2}(\hat{s})$ (top) and $T_{1}^{-1}(\hat{s})$ (in units of $\left.\mathrm{ps}^{-1} / \mathrm{at} \%\right)$ for $\hat{s}$ on the unit sphere for the case of a 10-layer W(001) film with a $\mathrm{W}$ adatom as scattering defect. The highest spin-relaxation values are found for $\hat{s}$ out of plane (here taken as the $z$ axis). 
maximum spin-relaxation rate of $T_{1}^{-1}=9.57 \mathrm{ps}^{-1} /$ at $\%$ is obtained for $\hat{s}$ out of plane; the minimum, $T_{1}^{-1}=7.55 \mathrm{ps}^{-1} / \mathrm{at} \%$, for $\hat{s}$ along the [110] axis. This yields an anisotropy of $\mathcal{A}\left[T_{1}^{-1}\right]=27 \%$. The map of $b^{2}(\hat{s})$ also shows a clear maximum for $\hat{s}$ out of plane with $b^{2}=0.294$ while in the [110] direction it has a value of $b^{2}=0.215$ giving an anisotropy of $\mathcal{A}\left[b^{2}\right]=$ $37 \%$. Evidently, there is no complete quantitative correlation between $b^{2}(\hat{s})$ and $T_{1}^{-1}(\hat{s})$, since the Elliott approximation is too crude in this case, but qualitatively the correlation is obvious. It should be noted that the in-plane variance of either $b^{2}(\hat{s})$ or $T_{1}^{-1}(\hat{s})$ is small (on the order of $2 \%$ ), owing to the high symmetry of the fourfold crystallographic axis. The anisotropy here for the thin films has a significantly higher value as compared to the anisotropy of $b^{2}$ of about $6 \%$ in bulk $\mathrm{W}$, as we have found previously, ${ }^{21}$ due to the reduced symmetry and to a great extent due to the surface states.

We can analyze the effect further by examining the Fermisurface-resolved $b_{\mathbf{k}}^{2}$ and $T_{1}^{-1}(\mathbf{k})$ [defined in Eq. (7)]. Figure 7 shows these quantities for $\hat{s} \|$ [001] (left) and $\hat{s} \|$ [110] (right). Both quantities $\left[b_{\mathbf{k}}^{2}\right.$ and $\left.T_{1}^{-1}(\mathbf{k})\right]$ show a symmetry compatible to the intersection of the symmetry operations leaving the
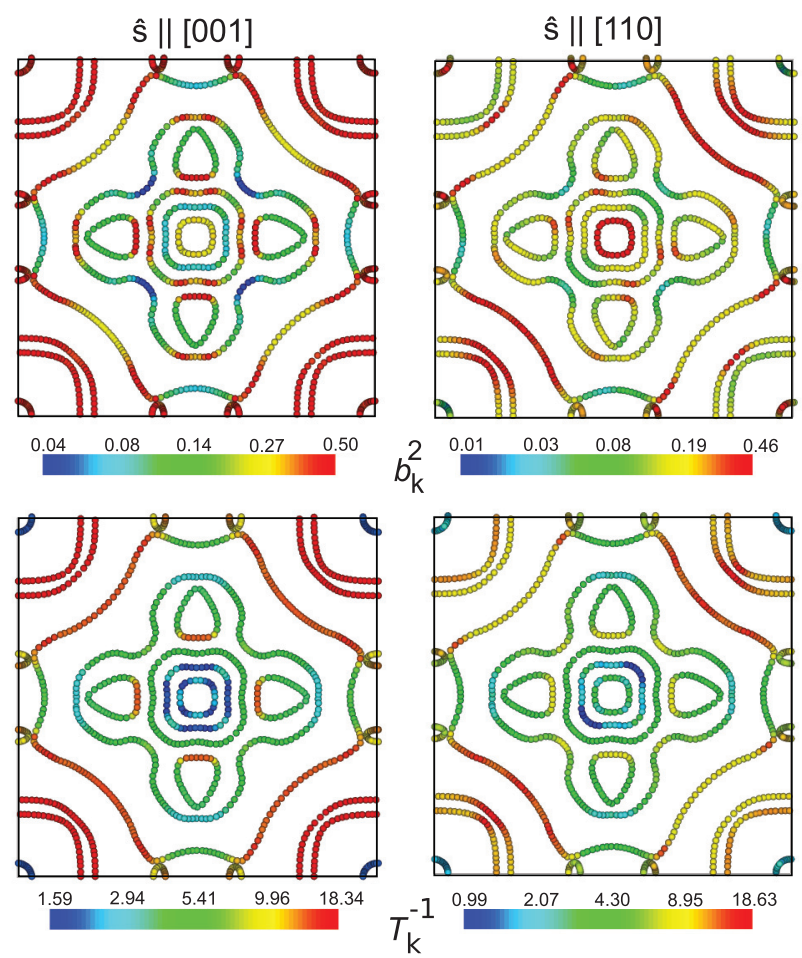

FIG. 7. (Color online) Distribution of the spin-mixing parameter $b_{\mathbf{k}}^{2}$ (top) and spin-relaxation rate $T_{1}^{-1}(\mathbf{k})$ (bottom) shown in a color code (in units of $\mathrm{ps}^{-1} / \mathrm{at} \%$ ) on the Fermi surface of a 10-layer $\mathrm{W}(001)$ film. For the calculation of $T_{1}^{-1}(\mathbf{k})$, scattering off $\mathrm{W}$ adatoms was considered. For the calculations shown in the left panels, the spin-quantization axis was taken in the [001] direction (i.e., perpendicular to the film), for the calculations shown in the right panels it was taken in the [110] direction (in-plane). The distributions on the Fermi surface are clearly compatible with the specific crystal symmetry operations that either leave $\hat{s}$ unchanged or result in $\hat{s} \rightarrow-\hat{s}$. Especially on the surface states, the values of $b_{\mathbf{k}}^{2}$ and $T_{1}^{-1}(\mathbf{k})$ are highest in the regions where the spin-polarization fields (see Fig. 5) are perpendicular to $\hat{s}$.
Fermi surface invariant and the operations leaving the SQA invariant plus the inversion. It is interesting to see that even though the system parameters are outside the prerequisites of the Elliott approximation $\left(b^{2}\right.$ is rather large and the scattering off a transition metal adatom cannot be considered weak), still a correlation between the "highs and lows" of $b_{\mathbf{k}}^{2}$ and $T_{1}^{-1}(\mathbf{k})$ is visible in the color code. Also, that the surface state regions show high values for both quantities when the spin-polarization fields (see Sec. IV and Fig. 5) are perpendicular to the SQA and lower values when they are parallel or antiparallel to the SQA. In this sense, and since there will be a part of the Rashba states with spin-polarization field perpendicular to the SQA no matter what the choice of the SQA is (compare with Fig. 5), the surface states are clearly "spin drains" for the system due to the spin polarization fields that are related to the Rashba effect; the worst case is $\hat{s} \|$ [001], when all spin-polarization fields are perpendicular to $\hat{s}$.

\section{REMARKS ON THE RELAXATION MECHANISM IN THE RASHBA STATES}

Finally, we wish to discuss the physical mechanism of spin relaxation in the presence of Rashba states. In semiconductors or semiconductor heterostructures, it is naturally assumed that the spin-polarization fields contribute to spin relaxation via the Dyakonov-Perel mechanism. ${ }^{11,12}$ There, an electron occupies a state at $\mathbf{k}$ that is split in energy by only a little according to an effective Hamiltonian $H=-\frac{1}{2} \boldsymbol{\Omega}_{\mathbf{k}} \cdot \boldsymbol{\sigma}$, thus in a semiclassical picture the electron spin precesses around the spin-orbit field $\boldsymbol{\Omega}_{\mathbf{k}}$ with a Larmor frequency $\left|\boldsymbol{\Omega}_{\mathbf{k}}\right|$, losing memory of its original direction before being scattered away (within this effective model the spin polarization field is parallel or antiparallel to the spin-orbit field $\boldsymbol{\Omega}_{\mathbf{k}}$ ). However, for this to happen it must be assumed that the electron wave packet has an energy spread larger than $\left|\boldsymbol{\Omega}_{\mathbf{k}}\right| .^{16,52}$ This is possible in semiconductors where $\left|\boldsymbol{\Omega}_{\mathbf{k}}\right|$ is usually small ${ }^{12}$ (of the order of $1 \mathrm{meV}$ or less, depending on temperature, doping concentration, etc.), as usually $\left|\boldsymbol{\Omega}_{\mathbf{k}}\right| \propto k$ with $\mathbf{k}$ very close to the conduction band minimum at $\Gamma$. As opposed to this, in metal surfaces the Rashba splitting $\Delta_{E_{\mathrm{F}}}$ of surface states at $E_{\mathrm{F}}$ can be large, of the order of $100 \mathrm{meV}$, e.g., $\Delta_{E_{\mathrm{F}}} \approx 200 \mathrm{meV}$ in $\mathrm{W}$ (see Fig. 4), $30 \mathrm{meV}$ in $\mathrm{Cu}(111),{ }^{16}$ and $150 \mathrm{meV}$ in $\mathrm{Au}(111) .{ }^{49,53}$ It is unlikely that a coherent wave packet excited by, e.g., an injected spin current or by microwave radiation in CESR should have such a large energy spread, activating the Dyakonov-Perel mechanism, except perhaps if prepared very precisely by an experiment targeting exactly this. Therefore we consider the Dyakonov-Perel mechanism not applicable in the case of the Rashba surface states of most metals, but we cannot exclude it for special cases, e.g., lighter metals with significantly weaker spin-orbit coupling or favorable band structure [e.g., in Ref. 53 we see that $\operatorname{Ag}(111)$ shows a splitting of only $\Delta_{E_{\mathrm{F}}}=2 \mathrm{meV}$ due to the shallow surface state].

\section{SUMMARY AND CONCLUSIONS}

In summary, we have investigated spin-relaxation physics of W(001) ultrathin films from first principles. We observe that the Elliott-Yafet parameter exhibits an even-odd oscillation 
with respect to the number of layers of the films, which stems from an even-odd oscillation in the surface electronic structure of the films at and near the Fermi energy. The oscillation is then inherited by the spin-relaxation rate that depends on the Elliott-Yafet parameter.

We have further identified the Rashba character and spinpolarization fields of the surface states and discussed how they contribute to the anisotropy of the spin-relaxation rate with respect to the relative orientation between the spinquantization axis and the crystallographic directions. The anisotropy values are much higher compared to those in bulk W.

We believe that our findings are not only particular to $\mathrm{W}(001)$ free standing films but are more general at least for transition metal films in the bcc structure and even when sandwiched between insulators. We base this speculation on three considerations: first, we performed calculations (not presented in the present paper) of the Elliott-Yafet parameter for Mo(001) films in the bcc structure and found basically the same oscillatory effect. Second, the existence of surface states is, in general, closely connected to the crystal structure; all bcc transition metals will show a dip in the density of states at the center of the $d$ band and in all cases the breaking of translational symmetry at the (001) surface will produce surface states of the character found here within the gap of the surface-projected bulk band structure. Third, the mechanism just stated is expected to produce interface states in the case that the film is in contact with an insulator, again with a spin-orbit splitting analogous to the Rashba splitting due to the interface asymmetry, even if the details of the interface band structure can be more complicated in this case.

As an outlook, we believe that it is worthwhile to investigate these effects in a broader family of ultrathin films. Such investigations are in progress and will be reported in a future publication.

\section{ACKNOWLEDGMENTS}

We would like to thank Gustav Bihlmayer for discussions on the Rashba effect and Martin Gradhand for discussions on the spin-flip scattering in films. This work was financially supported by the MO 1731/3-1 project and SPP 1538 SpinCaT program of the Deutsche Forschungsgemeinschaft, and the HGF-YIG NG-513 project of the Helmholtz Gemeinschaft. Computational resources were provided by the Jülich Supercomputing Center. *h.nguyen@fz-juelich.de

†ph.mavropoulos@fz-juelich.de

${ }^{1}$ I. Zutic, J. Fabian, and D. Sarma, Rev. Mod. Phys. 76, 323 (2004).

${ }^{2}$ T. Valet and A. Fert, Phys. Rev. B 48, 7099 (1993).

${ }^{3}$ J. Bass and W. P. Pratt Jr., J. Phys.: Condens. Matter 19, 183201 (2007).

${ }^{4}$ S. O. Valenzuela and M. Tinkham, Nature (London) 442, 176 (2006).

${ }^{5}$ T. Kimura, Y. Otani, T. Sato, S. Takahashi, and S. Maekawa, Phys. Rev. Lett. 98, 156601 (2007).

${ }^{6}$ T. Seki, Y. Hasegawa, S. Mitani, S. Takahashi, H. Imamura, S. Maekawa, J. Nitta, and K. Takanashi, Nat. Mater. 7, 125 (2008).

${ }^{7}$ F. Freimuth, S. Blügel, and Y. Mokrousov, Phys. Rev. Lett. 105, 246602 (2010).

${ }^{8}$ M. Gradhand, D. V. Fedorov, P. Zahn, and I. Mertig, Phys. Rev. B 81, 245109 (2010).

${ }^{9}$ E. Saitoh, M. Ueda, H. Miyajima, and G. Tatara, Appl. Phys. Lett. 88, 182509 (2006).

${ }^{10}$ V. P. Zhukov, E. V. Chulkov, and P. M. Echenique, Phys. Status Solidi A 205, 1296 (2008).

${ }^{11}$ M. I. Dyakonov and V. I. Perel, Fiz. Tverd. Tela 13, 3581 (1971); [Sov. Phys. Solid State 13, 3023 (1972)].

${ }^{12}$ J. Fabian, A. Matos-Abiague, C. Ertler, P. Stano, and I. Zutic, Acta Phys. Slovaca 57, 565 (2007).

${ }^{13}$ J. L. Cheng, M. W. Wu, and J. Fabian, Phys. Rev. Lett. 104, 016601 (2010).

${ }^{14}$ R. J. Elliott, Phys. Rev. 96, 266 (1954).

${ }^{15}$ Y. Yafet, Solid State Physics, edited by F. Seitz and D. Turnbull (Academic, NewYork, 1963), Vol. 14, p. 1.

${ }^{16}$ S. Heers, Ph.D. thesis, RWTH Aachen University, 2011; http://darwin.bth.rwth-aachen.de/opus3/volltexte/2011/3827/.
${ }^{17}$ S. LaShell, B. A. McDougall, and E. Jensen, Phys. Rev. Lett. 77, 3419 (1996).

${ }^{18}$ J. Henk, A. Ernst, and P. Bruno, Phys. Rev. B 68, 165416 (2003).

${ }^{19}$ H. A. Kramers, Proc. Amsterdam Acad. Sci. 33, 959 (1930).

${ }^{20}$ F. Pientka, M. Gradhand, D. V. Fedorov, I. Mertig, and B. L. Györffy, Phys. Rev. B 86, 054413 (2012).

${ }^{21}$ B. Zimmermann, P. Mavropoulos, S. Heers, N. H. Long, S. Blügel, and Y. Mokrousov, Phys. Rev. Lett. 109, 236603 (2012).

${ }^{22}$ Y. Mokrousov, H. Zhang, F. Freimuth, B. Zimmermann, N. H. Long, J. Weischenberg, I. Souza, P. Mavropoulos, and S. Blügel, J. Phys.: Condens. Matter 25, 163201 (2013).

${ }^{23}$ P. Monod and S. Schultz, J. Pysique (Paris) 43, 393 (1982).

${ }^{24}$ D. V. Fedorov, P. Zahn, M. Gradhand, and I. Mertig, Phys. Rev. B 77, 092406 (2008).

${ }^{25}$ V. L. Safonov, J. Appl. Phys. 91, 8653 (2002).

${ }^{26}$ A. Lyberatos, G. Yu, R. J. M. van de Veerdonk, and D. Weller, J. Appl. Phys. 91, 2236 (2002).

${ }^{27}$ A. L. Pankratov, S. N. Vdovichev, and I. M. Nefedov, Phys. Rev. B 78, 052401 (2008).

${ }^{28}$ S. L. Weng, E. W. Plummer, and T. Gustafsson, Phys. Rev. B 18, 1718 (1978).

${ }^{29}$ M. Posternak, H. Krakauer, A. J. Freeman, and D. D. Koelling, Phys. Rev. B 21, 5601 (1980).

${ }^{30}$ L. F. Mattheiss and D. R. Hamann, Phys. Rev. B 29, 5372 (1984).

${ }^{31}$ T. E. Felter, R. A. Barker, and P. J. Estrup, Phys. Rev. Lett. 38, 1138 (1977).

${ }^{32}$ M. K. Debe and D. A. King, Phys. Rev. Lett. 39, 708 (1977).

${ }^{33}$ D. Singh, S. H. Wei, and H. Krakauer, Phys. Rev. Lett 57, 3292 (1986).

${ }^{34}$ Z. Y. Li, R. N. Lamb, W. Allison, and R. F. Willis, Surf. Sci. 211-212, 931 (1989).

${ }^{35}$ G. A. Gaudin and M. J. G. Lee, Phys. Rev. B 49, 5575 (1994). 
${ }^{36}$ J. Almanstoötter, T. Firies, and B. Eberhard, J. App. Phys. 86, 325 (1999).

${ }^{37}$ P. Ferriani, K. von Bergmann, E. Y. Vedmedenko, S. Heinze, M. Bode, M. Heide, G. Bihlmayer, A. Kubetzka, A. Kubetzka, and S. Blügel, Phys. Rev. Lett. 101, 027201 (2008); 102, 019901(E) (2009).

${ }^{38}$ L. W. Swanson and L. C. Crouser, Phys. Rev. Lett. 16, 389 (1966)

${ }^{39}$ B. J. Waclawski and E. W. Plummer, Phys. Rev. Lett. 29, 783 (1972).

${ }^{40}$ S. H. Vosko, L. Wilk, and M. Nusair, Can. J. Phys. 58, 1200 (1980).

${ }^{41}$ N. Papanikolaou, R. Zeller, and P. H. Dederichs, J. Phys.: Condens. Matter 14, 2799 (2002); see also http://www.kkr-gf.org

${ }^{42}$ N. Stefanou and R. Zeller, J. Phys.: Condens. Matter 3, 7599 (1991); N. Stefanou, H. Akai, and R. Zeller, Comput. Phys. Commun. 60, 231 (1990).

${ }^{43}$ In Refs. 44 and 45, the fully relativistic KKR method is used, solving the Dirac equation during self-consistency, but in the atomic sphere approximation.

${ }^{44}$ M. Gradhand, M. Czerner, D. V. Fedorov, P. Zahn, B. Y. Yavorsky, L. Szunyogh, and I. Mertig, Phys. Rev. B 80, 224413 (2009).

${ }^{45}$ M. Gradhand, D. V. Fedorov, P. Zahn, and I. Mertig, Phys. Rev. B 81, 020403 (2010).
${ }^{46}$ D. Bauer, Ph.D. thesis, RWTH Aachen University, 2013.

${ }^{47}$ E. I. Rashba, Sov. Phys. Solid State 2, 1109 (1960).

${ }^{48}$ O. Krupin, G. Bihlmayer, K. Starke, S. Gorovikov, J. E. Prieto, K. Döbrich, S. Blügel, and G. Kaindl, Phys. Rev. B 71, 201403(R) (2005).

${ }^{49}$ M. Heide, G. Bihlmayer, P. Mavropoulos, A. Bringer, and S. Blügel, Psi-k scientific highlight No. 78 (2006), http://www.psi-k.org/newsletters/News_78/Highlight_78.pdf.

${ }^{50}$ A. Eiguren and C. Ambrosch-Draxl, New J. Phys. 11, 013056 (2009).

${ }^{51}$ The approach was inspired from the work on giant Rashba splitting in Bi ultrathin films by T. Hirahara, K. Miyamoto, A. Kimura, Y. Niinuma, G. Bihlmayer, E. V. Chulkov, T. Nagao, I. Matsuda, S. Qiao, K. Shimada, H. Namatame, M. Taniguchi, and. S. Hasegawa, New J. Phys. 10, 083038 (2008).

${ }^{52}$ P. Mavropoulos, Spintronics: From GMR to Quantum Information, Lecture Notes of the 40th Spring School, edited by S. Blügel, D. Bürgler, M. Morgenstern, C. M. Schneider, and R. Waser (Forschungszentrum Jülich, Jülich, 2009), Chap. B6.

${ }^{53}$ G. Nicolay, F. Reinert, S. Hüfner, and P. Blaha, Phys. Rev. B 65, 033407 (2001). 